\title{
Approximating a common point of fixed points of a pseudocontractive mapping and zeros of sum of monotone mappings
}

Naseer Shahzad ${ }^{1 *}$ and Habtu Zegeye ${ }^{2}$

"Correspondence:

nshahzad@kau.edu.sa

'Department of Mathematics, King

Abdulaziz University, P.O. Box 80203,

Jeddah, 21589, Saudi Arabia

Full list of author information is

available at the end of the article

\begin{abstract}
Let $C$ be a closed and convex subset of a real Hilbert space $H$. Let $T$ be a Lipschitzian pseudocontractive mapping of $C$ into itself, $A$ be a $\gamma$-inverse strongly monotone mapping of $C$ into $H$ and let $B$ be a maximal monotone operator on $H$ such that the domain of $B$ is included in $C$. We introduce an iteration scheme for finding a minimum-norm point of $F(T) \cap(A+B)^{-1}(0)$. Application to a common element of the set of fixed points of a Lipschitzian pseudocontractive and solutions of variational inequality for $\alpha$-inverse strongly monotone mappings is included. Our theorems improve and unify most of the results that have been proved in this direction for this important class of nonlinear mappings. To the best of our knowledge, approximating a common fixed point of pseudocontractive mappings with explicit scheme has not been possible and our result is even the first result that states the solution of a variational inequality in the set of fixed points of pseudocontractive mappings. Our scheme which is explicit is the best to use for the problem under consideration.

MSC: $47 \mathrm{H} 05 ; 47 \mathrm{H} 09 ; 47 J 25$
\end{abstract}

Keywords: fixed points; monotone mappings; pseudocontractive mappings; strong convergence

\section{Introduction}

Let $C$ be a closed convex subset of a real Hilbert space $H$. A mapping $T: C \rightarrow H$ is called $a$ contraction mapping if there exists $L \in[0,1)$ such that $\|T x-T y\| \leq L\|x-y\|$ for all $x, y \in C$. If $L=1$ then $T$ is called nonexpansive. $T$ is called quasi-nonexpansive if $\|T x-T p\| \leq\|x-p\|$ for all $x \in C$ and $p \in F(T)$, where $F(T):=\{x \in C: T x=x\}$, the set of fixed points of $T$. A mapping $T$ is called $\gamma$-strictly pseudocontractive [1] if and only if there exists $\gamma \in[0,1)$ such that

$$
\|T x-T y\|^{2} \leq\|x-y\|^{2}+\gamma\|(I-T) x-(I-T) y\|^{2}, \quad \text { for all } x, y \in C,
$$

and $T$ is called pseudocontractive if

$$
\|T x-T y\|^{2} \leq\|x-y\|^{2}+\|(I-T) x-(I-T) y\|^{2}, \quad \text { for all } x, y \in C,
$$

O2014 Shahzad and Zegeye; licensee Springer. This is an Open Access article distributed under the terms of the Creative Commons Attribution License (http://creativecommons.org/licenses/by/2.0), which permits unrestricted use, distribution, and reproduction in any medium, provided the original work is properly cited. 
where $I$ is the identity mapping. We note that inequalities (1.1) and (1.2) can be equivalently written as

$$
\langle x-y, T x-T y\rangle \leq\|x-y\|^{2}-\lambda\|(I-T) x-(I-T) y\|^{2}
$$

for some $\lambda>0$, and

$$
\langle x-y, T x-T y\rangle \leq\|x-y\|^{2}, \quad \text { for all } x, y \in C,
$$

respectively.

Clearly, the class of nonexpansive mappings is a subset of the class of $\gamma$-strictly pseudocontractive mappings and the class of $\gamma$-strictly pseudocontractive is contained in the class of pseudocontractive mappings. Moreover, this inclusion is strict due to the following example in [2].

Take $X=\mathbb{R}^{2}, B=\left\{x \in \mathbb{R}^{2}:\|x\| \leq 1\right\}, B_{1}=\left\{x \in B:\|x\| \leq \frac{1}{2}\right\}, B_{2}=\left\{x \in B: \frac{1}{2} \leq\|x\| \leq 1\right\}$. If $x=(a, b) \in X$ we define $x \perp$ to be $(b,-a) \in X$. Define $T: B \rightarrow B$ by

$$
T x= \begin{cases}x+x \perp, & \text { if } x \in B_{1}, \\ \frac{x}{\|x\|}-x+x \perp, & \text { if } x \in B_{2} .\end{cases}
$$

Then $T$ is a Lipschitzian and pseudocontractive mapping but not a strictly pseudocontractive mapping.

Closely related to the class of pseudocontractive mappings is the class of monotone mappings. A mapping $A: C \rightarrow H$ is called monotone if

$$
\langle x-y, A x-A y\rangle \geq 0, \quad \text { for all } x, y \in C,
$$

and $A$ is called $\gamma$-inverse strongly monotone if there exists a positive real number $\gamma$ such that

$$
\langle x-y, A x-A y\rangle \geq \gamma\|A x-A y\|^{2}, \quad \text { for all } x, y \in C .
$$

If $A$ is $\gamma$-inverse strongly monotone, then inequality (1.7) implies that $A$ is Lipschitzian with constant $L:=\frac{1}{\gamma}$, that is, $\|A x-A y\| \leq \frac{1}{\gamma}\|x-y\|$, for all $x, y \in C$.

We remark the $T$ is $\gamma$-strictly pseudocontractive if and only if $A:=(I-T)$ is $\gamma$-inverse strongly monotone and $T$ is pseudocontractive if and only if $A:=(I-T)$ is monotone. Clearly, the class of monotone mappings includes the class of $\gamma$-inverse strongly monotone mappings. We note that the inclusion is proper. This can be seen from the example in [2]. Take $A:=(I-T)$, where $T$ is as in (1.5). Then we see that $A$ is monotone but not $\gamma$-inverse strongly monotone as $T$ is not strictly pseudocontractive.

A mapping $A$ is called maximal monotone if it is monotone and $\mathcal{R}(I+r A)$, the range of $(I+r A)$, is $H$ for all $r>0$. If $A$ is maximal monotone, then to each $r>0$ and $x \in H$, there corresponds a unique element $x_{r} \in D(A)$ satisfying

$$
x \in x_{r}+r A x_{r} .
$$

We denote the resolvent of $A$ by $J_{r} x=x_{r}$. That is, $J_{r}=(I+r A)^{-1}$ for all $r>0$. If $A$ is monotone then $J_{r}:=(I+r A)^{-1}$ is nonexpansive single valued mapping from $\mathcal{R}(I+r A)$ into $D(A)$ and $F\left(J_{r}\right)=N(A)$ (see [3]). 
It is now well known (see e.g. [4]) that if $A$ is monotone then the solutions of the equation $A x=0$ correspond to the equilibrium points of some evolution systems. Consequently, considerable research efforts, especially within the past 20 years or so, have been devoted to iterative methods for approximating the zeros of monotone mapping $A$ or fixed point of pseudocontractive mapping $T$ (see, for example, [5-11]).

Let $A$ be a nonlinear mapping on $H$. Consider the problem of finding

$$
u \in C \text { such that } 0 \in A u \text {. }
$$

When $A$ is a maximal monotone mapping, a well-known methods for solving (1.8) is the proximal point algorithm: $x_{1}=x \in H$, and

$$
x_{n+1}=J_{r_{n}} x_{n}, \quad n=1,2,3, \ldots,
$$

where $J_{r_{n}}=\left(I+r_{n} A\right)^{-1}$ and $\left\{r_{n}\right\} \subset(0, \infty)$, then Rockafellar [12] (also see [13]) proved that the sequence $\left\{x_{n}\right\}$ converges weakly to an element of $A^{-1}(0)$.

In [14], Kamimura and Takahashi investigated the problem of finding a zero point of a maximal monotone mapping by considering the following iterative algorithm:

$$
x_{0} \in H, \quad x_{n+1}=\alpha_{n} x_{n}+\left(1-\alpha_{n}\right) J_{\lambda_{n}} x_{n}, \quad n=0,1, \ldots,
$$

where $\left\{\alpha_{n}\right\}$ is a sequence in $(0,1),\left\{\lambda_{n}\right\}$ is a positive sequence, $A: H \rightarrow H$ is a maximal monotone, and $J_{\lambda_{n}}=\left(I+\lambda_{n} A\right)^{-1}$. They showed that the sequence $\left\{x_{n}\right\}$ generated by (1.9) converges weakly to some $z \in A^{-1}(0)$ in the framework of real Hilbert spaces, provided that the control sequences satisfy some restrictions.

Let $C$ be a nonempty, closed and convex subset of $H$ and $A: C \rightarrow H$ be a nonlinear mapping. The variational inequality problem which was introduced and studied by Stampacchia [15] is to:

$$
\text { find } u \in C \text { such that }\langle A u, v-u\rangle \geq 0, \quad \forall v \in C \text {. }
$$

The set of solutions of the variational inequality problem is denoted by $\operatorname{VI}(C, A)$.

Variational inequality theory has emerged as an important tool in studying a wide class of numerous problems in physics, optimization, variational inequalities, minimax problems, and the Nash equilibrium problems in noncooperative games (see, for instance, [1622]).

In [23], Takahashi and Toyoda investigated the problem of finding a common point of solutions of the variational inequality problem (1.10) for $A: C \rightarrow H$ a $\gamma$-inverse strongly monotone mapping and fixed points of a nonexpansive mapping $T: C \rightarrow C$ by considering the following iterative algorithm:

$$
x_{0} \in H, \quad x_{n+1}=\alpha_{n} x_{n}+\left(1-\alpha_{n}\right) T P_{C}\left(x_{n}-\lambda_{n} A x_{n}\right), \quad n=0,1, \ldots
$$

where $\left\{\alpha_{n}\right\}$ is a sequence in $(0,1),\left\{\lambda_{n}\right\}$ is a positive sequence. They proved that the sequence $\left\{x_{n}\right\}$ generated by (1.11) converges weakly to some $z \in \operatorname{VI}(C, A) \cap F(T)$ provided that the control sequences satisfy some restrictions. 
It is worth to mention that the methods studied above give weak convergence theorems in the framework of Hilbert spaces.

Regarding iterative method for a common point of fixed points of nonexpansive and zeros of sum of two monotone mappings, Takahashi et al. [24] proved the following theorem.

Theorem TT [24] Let C be a nonempty closed and convex subset of a real Hilbert space $H$. Let $A$ be a $\gamma$-inverse strongly monotone mapping of $C$ into $H$ and let $B$ be a maximal monotone mapping on $H$ such that the domain of $B$ is included in $C$. Let $J_{\lambda}=(I+\lambda B)^{-1}$ be the resolvent of $B$, for $\lambda>0$, and let $T$ be a nonexpansive mapping of $C$ into itself such that $F(T) \cap(A+B)^{-1} \neq \emptyset$. Let $x_{1}=x \in C$ and let $\left\{x_{n}\right\} \subset C$ be a sequence generated by

$$
x_{n+1}=\beta_{n} x_{n}+\left(1-\beta_{n}\right) T\left(\alpha_{n} x+\left(1-\alpha_{n}\right) J_{\lambda_{n}}\left(x_{n}-\lambda_{n} A x_{n}\right)\right), \quad n=1,2, \ldots,
$$

where $\left\{\lambda_{n}\right\},\left\{\beta_{n}\right\}$ and $\left\{\alpha_{n}\right\}$ satisfy certain conditions. Then $\left\{x_{n}\right\}$ converges strongly to a point of $F(T) \cap(A+B)^{-1}(0)$.

For other related results, we refer to [25-30].

A natural question arises: can we obtain an iterative scheme which converges strongly to a common point of fixed points of the pseudocontractive mapping $T$ and zeros of two monotone mappings?

It is our purpose in this paper to introduce an iterative scheme which converges strongly to a common minimum-norm point of fixed points of a Lipschitzian pseudocontractive mapping and zeros of sum of two monotone mappings. Application to a common element of the set of fixed points of a Lipschitzian pseudocontractive mapping and solutions of variational inequality for $\gamma$-inverse strongly monotone mapping is included. The results obtained in this paper improve and extend the results of Kamimura and Takahashi [14], Takahashi and Toyoda [23], Takahashi et al. [24] and some other results in this direction.

\section{Preliminaries}

In what follows we shall make use of the following lemmas.

Lemma 2.1 [31] Let $C$ be a convex subset of a real Hilbert space $H$. Let $x \in H$. Then $x_{0}=$ $P_{C} x$ if and only if

$$
\left\langle z-x_{0}, x-x_{0}\right\rangle \leq 0, \quad \forall z \in C
$$

We also remark that in a real Hilbert space $H$, the following identity holds:

$$
\|x+y\|^{2} \leq\|x\|^{2}+2\langle y, x+y\rangle, \quad \forall x, y \in H .
$$

Lemma 2.2 [32] Let $\left\{a_{n}\right\}$ be a sequence of nonnegative real numbers satisfying the following relation:

$$
a_{n+1} \leq\left(1-\alpha_{n}\right) a_{n}+\alpha_{n} \delta_{n}, \quad n \geq n_{0},
$$

where $\left\{\alpha_{n}\right\} \subset(0,1)$ and $\left\{\delta_{n}\right\} \subset R$ satisfying the following conditions: $\lim _{n \rightarrow \infty} \alpha_{n}=0$, $\sum_{n=1}^{\infty} \alpha_{n}=\infty$, and $\lim \sup _{n \rightarrow \infty} \delta_{n} \leq 0$. Then $\lim _{n \rightarrow \infty} a_{n}=0$. 
Lemma 2.3 [33] Let $H$ be a real Hilbert space, $C$ a closed convex subset of $H$ and $T: C \rightarrow C$ be a continuous pseudocontractive mapping, then

(i) $F(T)$ is closed convex subset of $C$;

(ii) $(I-T)$ is demiclosed at zero, i.e., if $\left\{x_{n}\right\}$ is a sequence in $C$ such that $x_{n} \rightarrow x$ and $T x_{n}-x_{n} \rightarrow 0$, as $n \rightarrow \infty$, then $x=T(x)$.

Lemma 2.4 [34] Let $\left\{a_{n}\right\}$ be sequences of real numbers such that there exists a subsequence $\left\{n_{i}\right\}$ of $\{n\}$ such that $a_{n_{i}}<a_{n_{i}+1}$ for all $i \in \mathbb{N}$. Then there exists a nondecreasing sequence $\left\{m_{k}\right\} \subset \mathbb{N}$ such that $m_{k} \rightarrow \infty$ and the following properties are satisfied by all (sufficiently large) numbers $k \in \mathbb{N}$ :

$$
a_{m_{k}} \leq a_{m_{k}+1} \text { and } a_{k} \leq a_{m_{k}+1}
$$

In fact, $m_{k}=\max \left\{j \leq k: a_{j}<a_{j+1}\right\}$.

Lemma 2.5 [35] Let $H$ be a real Hilbert space. Then for all $x_{i} \in H$ and $\alpha_{i} \in[0,1]$ for $i=$ $1,2, \ldots, n$ such that $\alpha_{1}+\alpha_{2}+\cdots+\alpha_{n}=1$ the following equality holds:

$$
\left\|\alpha_{0} x_{0}+\alpha_{1} x_{1}+\cdots+\alpha_{n} x_{n}\right\|^{2}=\sum_{i=0}^{n} \alpha_{i}\left\|x_{i}\right\|^{2}-\sum_{0 \leq i, j \leq n} \alpha_{i} \alpha_{j}\left\|x_{i}-x_{j}\right\|^{2} .
$$

Lemma 2.6 [36] Let $C$ be a nonempty closed and convex subset of a real Hilbert space $H$. Let $A: C \rightarrow E$ be $\gamma$-inverse strongly monotone mapping. Then, for $0<\mu<2 \gamma$, the mapping $A_{\mu} x:=(x-\mu A x)$ is nonexpansive.

Lemma 2.7 [37] Let $H$ be a Hilbert space. Let $A: D(A) \subseteq H \rightarrow 2^{H}$ and $B: D(B) \subseteq H \rightarrow$ $2^{H}$ be maximal monotone mappings. Suppose that $D(A) \cap \operatorname{int} D(B) \neq \emptyset$. Then $A+B$ is a maximal monotone mapping.

\section{Main result}

Theorem 3.1 Let $C$ be a nonempty, closed and convex subset of a real Hilbert space $H$. Let $T: C \rightarrow C$ be a Lipschitzian pseudocontractive mapping with Lipschitz constant $L$. Let $A: C \rightarrow H$ be a $\gamma$-inverse strongly monotone mapping and $B$ be a maximal monotone mapping on $H$ such that the domain of $B$ is subset of $C$. Assume that $\mathcal{F}=F(T) \cap(A+B)^{-1}(0)$ is nonempty. Let $\left\{x_{n}\right\}$ be the sequence generated from an arbitrary $x_{0} \in C$ by

$$
\left\{\begin{array}{l}
y_{n}=\left(1-\beta_{n}\right) x_{n}+\beta_{n} T x_{n} \\
x_{n+1}=P_{C}\left[\left(1-\alpha_{n}\right)\left(\theta_{n} x_{n}+\delta_{n} T y_{n}+\gamma_{n} T_{\lambda_{n}} x_{n}\right)\right]
\end{array}\right.
$$

where $T_{\lambda_{n}}\left(x_{n}\right):=\left(I+\lambda_{n} B\right)^{-1}\left(I-\lambda_{n} A\right) x_{n}$ and $\left\{\lambda_{n}\right\} \subset(a, b) \subset(a, 2 \gamma),\left\{\theta_{n}\right\},\left\{\delta_{n}\right\},\left\{\gamma_{n}\right\} \subset(c, d) \subset$ $(0,1),\left\{\alpha_{n}\right\} \subset(0, e) \subset(0,1)$, for some $a, b, c, d, e>0$, satisfying the following conditions: (i) $\theta_{n}+\delta_{n}+\gamma_{n}=1$, (ii) $\lim _{n \rightarrow \infty} \alpha_{n}=0, \sum \alpha_{n}=\infty$; (iii) $\delta_{n}+\gamma_{n} \leq \beta_{n} \leq \beta<\frac{1}{\sqrt{1+L^{2}}+1}, \forall n \geq 1$. Then $\left\{x_{n}\right\}$ converges strongly to the minimum-norm point $x^{*}$ of $\mathcal{F}$.

Proof From Lemma 2.6 and the fact that $J_{\lambda_{n}}$ is nonexpansive we see that $T_{\lambda_{n}}$ is nonexpansive. Let $p \in \mathcal{F}$. Then from (3.1), (1.2), Lemma 2.5 and using the fact that $p=T_{\lambda_{n}}(p)$ we 
have

$$
\begin{aligned}
\left\|x_{n+1}-p\right\|^{2}= & \left\|P_{C}\left[\left(1-\alpha_{n}\right)\left(\theta_{n} x_{n}+\delta_{n} T y_{n}+\gamma_{n} T_{\lambda_{n}} x_{n}\right)\right]-p\right\|^{2} \\
\leq & \left\|\left(1-\alpha_{n}\right)\left(\theta_{n} x_{n}+\delta_{n} T y_{n}+\gamma_{n} T_{\lambda_{n}} x_{n}\right)-p\right\|^{2} \\
\leq & \alpha_{n}\|p\|^{2}+\left(1-\alpha_{n}\right)\left\|\theta_{n}\left(x_{n}-p\right)+\delta_{n}\left(T y_{n}-p\right)+\gamma_{n}\left(T_{\lambda_{n}} x_{n}-p\right)\right\|^{2} \\
\leq & \alpha_{n}\|p\|^{2}+\left(1-\alpha_{n}\right)\left[\theta_{n}\left\|x_{n}-p\right\|^{2}+\delta_{n}\left\|T y_{n}-p\right\|^{2}\right. \\
& \left.+\gamma_{n}\left\|T_{\lambda_{n}} x_{n}-p\right\|^{2}\right]-\left(1-\alpha_{n}\right) \delta_{n} \theta_{n}\left\|T y_{n}-x_{n}\right\|^{2} \\
& -\left(1-\alpha_{n}\right) \theta_{n} \gamma_{n}\left\|T_{\lambda_{n}} x_{n}-x_{n}\right\|^{2} \\
\leq & \alpha_{n}\|p\|^{2}+\left(1-\alpha_{n}\right)\left(\theta_{n}+\gamma_{n}\right)\left\|x_{n}-p\right\|^{2}+\left(1-\alpha_{n}\right) \delta_{n}\left\|T y_{n}-p\right\|^{2} \\
& -\left(1-\alpha_{n}\right) \delta_{n} \theta_{n}\left\|T y_{n}-x_{n}\right\|^{2}-\left(1-\alpha_{n}\right) \theta_{n} \gamma_{n}\left\|T_{\lambda_{n}} x_{n}-x_{n}\right\|^{2}
\end{aligned}
$$

and hence

$$
\begin{aligned}
\left\|x_{n+1}-p\right\|^{2} \leq & \alpha_{n}\|p\|^{2}+\left(1-\alpha_{n}\right)\left(\theta_{n}+\gamma_{n}\right)\left\|x_{n}-p\right\|^{2}+\left(1-\alpha_{n}\right) \delta_{n}\left[\left\|y_{n}-p\right\|^{2}\right. \\
& \left.+\left\|y_{n}-T y_{n}\right\|^{2}\right]-\left(1-\alpha_{n}\right) \delta_{n} \theta_{n}\left\|T y_{n}-x_{n}\right\|^{2} \\
& -\left(1-\alpha_{n}\right) \theta_{n} \gamma_{n}\left\|T_{\lambda_{n}} x_{n}-x_{n}\right\|^{2} \\
= & \alpha_{n}\|p\|^{2}+\left(1-\alpha_{n}\right)\left(\theta_{n}+\gamma_{n}\right)\left\|x_{n}-p\right\|^{2}+\left(1-\alpha_{n}\right) \delta_{n}\left\|y_{n}-p\right\|^{2} \\
& +\left(1-\alpha_{n}\right) \delta_{n}\left\|y_{n}-T y_{n}\right\|^{2}-\left(1-\alpha_{n}\right) \delta_{n} \theta_{n}\left\|T y_{n}-x_{n}\right\|^{2} \\
& -\left(1-\alpha_{n}\right) \theta_{n} \gamma_{n}\left\|T_{\lambda_{n}} x_{n}-x_{n}\right\|^{2} .
\end{aligned}
$$

In addition, from (3.1), Lemma 2.5, and (1.2) we get

$$
\begin{aligned}
\left\|y_{n}-p\right\|^{2}= & \left\|\left(1-\beta_{n}\right)\left(x_{n}-p\right)+\beta_{n}\left(T x_{n}-p\right)\right\|^{2} \\
= & \left(1-\beta_{n}\right)\left\|x_{n}-p\right\|^{2}+\beta_{n}\left\|T x_{n}-p\right\|^{2} \\
& -\beta_{n}\left(1-\beta_{n}\right)\left\|x_{n}-T x_{n}\right\|^{2} \\
\leq & \left(1-\beta_{n}\right)\left\|x_{n}-p\right\|^{2}+\beta_{n}\left[\left\|x_{n}-p\right\|^{2}+\left\|x_{n}-T x_{n}\right\|^{2}\right] \\
& -\beta_{n}\left(1-\beta_{n}\right)\left\|x_{n}-T x_{n}\right\|^{2} \\
= & \left\|x_{n}-p\right\|^{2}+\beta_{n}^{2}\left\|x_{n}-T x_{n}\right\|^{2}
\end{aligned}
$$

and

$$
\begin{aligned}
\left\|y_{n}-T y_{n}\right\|^{2}= & \left\|\left(1-\beta_{n}\right)\left(x_{n}-T y_{n}\right)+\beta_{n}\left(T x_{n}-T y_{n}\right)\right\|^{2} \\
= & \left(1-\beta_{n}\right)\left\|x_{n}-T y_{n}\right\|^{2}+\beta_{n}\left\|T x_{n}-T y_{n}\right\|^{2} \\
& -\beta_{n}\left(1-\beta_{n}\right)\left\|x_{n}-T x_{n}\right\|^{2} \\
\leq & \left(1-\beta_{n}\right)\left\|x_{n}-T y_{n}\right\|^{2}+\beta_{n} L^{2}\left\|x_{n}-y_{n}\right\|^{2} \\
& -\beta_{n}\left(1-\beta_{n}\right)\left\|x_{n}-T x_{n}\right\|^{2} \\
= & \left(1-\beta_{n}\right)\left\|x_{n}-T y_{n}\right\|^{2}+\beta_{n}^{3} L^{2}\left\|x_{n}-T x_{n}\right\|^{2}
\end{aligned}
$$




$$
\begin{aligned}
& -\beta_{n}\left(1-\beta_{n}\right)\left\|x_{n}-T x_{n}\right\|^{2} \\
= & \left(1-\beta_{n}\right)\left\|x_{n}-T y_{n}\right\|^{2}-\beta_{n}\left(1-L^{2} \beta_{n}^{2}-\beta_{n}\right)\left\|x_{n}-T x_{n}\right\|^{2} .
\end{aligned}
$$

Substituting (3.3) and (3.4) into (3.2) we obtain

$$
\begin{aligned}
\left\|x_{n+1}-p\right\|^{2} \leq & \alpha_{n}\|p\|^{2}+\left(1-\alpha_{n}\right)\left(\theta_{n}+\gamma_{n}\right)\left\|x_{n}-p\right\|^{2}+\left(1-\alpha_{n}\right) \delta_{n}\left[\left\|x_{n}-p\right\|^{2}\right. \\
& \left.+\beta_{n}^{2}\left\|x_{n}-T x_{n}\right\|^{2}\right]+\left(1-\alpha_{n}\right) \delta_{n}\left[\left(1-\beta_{n}\right)\left\|x_{n}-T y_{n}\right\|^{2}\right. \\
& \left.-\beta_{n}\left(1-L^{2} \beta_{n}^{2}-\beta_{n}\right)\left\|x_{n}-T x_{n}\right\|^{2}\right]-\left(1-\alpha_{n}\right) \delta_{n} \theta_{n}\left\|T y_{n}-x_{n}\right\|^{2} \\
& -\left(1-\alpha_{n}\right) \theta_{n} \gamma_{n}\left\|T_{\lambda_{n}} x_{n}-x_{n}\right\|^{2} \\
= & \alpha_{n}\|p\|^{2}+\left(1-\alpha_{n}\right)\left\|x_{n}-p\right\|^{2}-\left(1-\alpha_{n}\right) \delta_{n} \beta_{n}\left(1-\left(L^{2} \beta_{n}^{2}+2 \beta_{n}\right)\right) \\
& \times\left\|x_{n}-T x_{n}\right\|^{2}+\left(1-\alpha_{n}\right) \delta_{n}\left(1-\theta_{n}-\beta_{n}\right)\left\|T y_{n}-x_{n}\right\|^{2} \\
& -\left(1-\alpha_{n}\right) \theta_{n} \gamma_{n}\left\|T_{\lambda_{n}} x_{n}-x_{n}\right\|^{2},
\end{aligned}
$$

and hence

$$
\begin{aligned}
\left\|x_{n+1}-p\right\|^{2} \leq & \alpha_{n}\|p\|^{2}+\left(1-\alpha_{n}\right)\left\|x_{n}-p\right\|^{2}-\left(1-\alpha_{n}\right) \delta_{n} \beta_{n} \\
& \times\left(1-\left(L^{2} \beta_{n}^{2}+2 \beta_{n}\right)\right)\left\|x_{n}-T x_{n}\right\|^{2} \\
& +\left(1-\alpha_{n}\right) \delta_{n}\left(\delta_{n}+\gamma_{n}-\beta_{n}\right)\left\|T y_{n}-x_{n}\right\|^{2} \\
& -\left(1-\alpha_{n}\right) \theta_{n} \gamma_{n}\left\|T_{\lambda_{n}} x_{n}-x_{n}\right\|^{2} .
\end{aligned}
$$

Now, from (iii) of the hypotheses we have

$$
1-2 \beta_{n}-L^{2} \beta_{n}^{2} \geq 1-2 \beta-L^{2} \beta^{2}>0
$$

and

$$
\left(\delta_{n}+\gamma_{n}\right)-\beta_{n} \leq 0, \quad \text { for all } n \geq 1
$$

Thus, inequality (3.5) implies that

$$
\left\|x_{n+1}-p\right\|^{2} \leq \alpha_{n}\|p\|^{2}+\left(1-\alpha_{n}\right)\left\|x_{n}-p\right\|^{2} .
$$

Thus, by induction,

$$
\left\|x_{n+1}-p\right\|^{2} \leq \max \left\{\|p\|^{2},\left\|x_{0}-p\right\|^{2}\right\}, \quad \forall n \geq 0,
$$

which implies that $\left\{x_{n}\right\}$ and hence $\left\{y_{n}\right\}$ are bounded.

Let $w_{n}:=\left(1-\alpha_{n}\right)\left(\theta_{n} x_{n}+\delta_{n} T y_{n}+\gamma_{n} T_{\lambda_{n}} x_{n}\right)$. Then we see that $x_{n+1}=P_{C} w_{n}$. Let $x^{*}=P_{\mathcal{F}}(0)$. Then, using (3.1), (2.1) and following the methods used to get (3.5), we obtain

$$
\begin{aligned}
\left\|x_{n+1}-x^{*}\right\|^{2} & =\left\|P_{C}\left[\left(1-\alpha_{n}\right)\left(\theta_{n} x_{n}+\delta_{n} T y_{n}+\gamma_{n} T_{\lambda_{n}} x_{n}\right)\right]-x^{*}\right\|^{2} \\
& \leq\left\|\alpha_{n}\left(-x^{*}\right)+\left(1-\alpha_{n}\right)\left[\theta_{n} x_{n}+\delta_{n} T y_{n}+\gamma_{n} T_{\lambda_{n}} x_{n}-x^{*}\right]\right\|^{2}
\end{aligned}
$$




$$
\begin{aligned}
\leq & \left(1-\alpha_{n}\right)\left\|\delta_{n} T y_{n}+\theta_{n} x_{n}+\gamma_{n} T_{\lambda_{n}} x_{n}-x^{*}\right\|^{2} \\
& +2 \alpha_{n}\left\langle-x^{*}, w_{n}-x^{*}\right\rangle \\
\leq & \left(1-\alpha_{n}\right) \delta_{n}\left\|T y_{n}-x^{*}\right\|^{2}+\left(1-\alpha_{n}\right) \theta_{n}\left\|x_{n}-x^{*}\right\|^{2} \\
& +\left(1-\alpha_{n}\right) \gamma_{n}\left\|T_{\lambda_{n}} x_{n}-x^{*}\right\|^{2}-\left(1-\alpha_{n}\right) \theta_{n} \delta_{n}\left\|T y_{n}-x_{n}\right\|^{2} \\
& -\left(1-\alpha_{n}\right) \theta_{n} \gamma_{n}\left\|T_{\lambda_{n}} x_{n}-x_{n}\right\|^{2}+2 \alpha_{n}\left\langle-x^{*}, w_{n}-x^{*}\right\rangle
\end{aligned}
$$

and so

$$
\begin{aligned}
\left\|x_{n+1}-x^{*}\right\|^{2} \leq & \left(1-\alpha_{n}\right) \delta_{n}\left[\left\|y_{n}-x^{*}\right\|^{2}+\left\|y_{n}-T y_{n}\right\|^{2}\right] \\
& +\left(1-\alpha_{n}\right)\left(\theta_{n}+\gamma_{n}\right)\left\|x_{n}-x^{*}\right\|^{2} \\
& -\left(1-\alpha_{n}\right) \theta_{n} \delta_{n}\left\|T y_{n}-x_{n}\right\|^{2} \\
& -\left(1-\alpha_{n}\right) \theta_{n} \gamma_{n}\left\|T_{\lambda_{n}} x_{n}-x_{n}\right\|^{2}+2 \alpha_{n}\left\langle-x^{*}, w_{n}-x^{*}\right\rangle \\
\leq & \left(1-\alpha_{n}\right) \delta_{n}\left[\left\|x_{n}-x^{*}\right\|^{2}+\beta_{n}^{2}\left\|x_{n}-T x_{n}\right\|^{2}\right] \\
& +\left(1-\alpha_{n}\right) \delta_{n}\left[\left(1-\beta_{n}\right)\left\|x_{n}-T y_{n}\right\|^{2}-\beta_{n}\left(1-L^{2} \beta_{n}^{2}-\beta_{n}\right)\right. \\
& \left.\times\left\|x_{n}-T x_{n}\right\|^{2}\right]+\left(1-\alpha_{n}\right)\left(\theta_{n}+\gamma_{n}\right)\left\|x_{n}-x^{*}\right\|^{2} \\
& -\left(1-\alpha_{n}\right) \theta_{n} \delta_{n}\left\|T y_{n}-x_{n}\right\|^{2}-\left(1-\alpha_{n}\right) \theta_{n} \gamma_{n}\left\|T_{\lambda_{n}} x_{n}-x_{n}\right\|^{2} \\
& +2 \alpha_{n}\left(-x^{*}, w_{n}-x^{*}\right\rangle,
\end{aligned}
$$

which implies that

$$
\begin{aligned}
\left\|x_{n+1}-x^{*}\right\|^{2} \leq & \left(1-\alpha_{n}\right)\left\|x_{n}-x^{*}\right\|^{2}-\left(1-\alpha_{n}\right) \delta_{n} \beta_{n}\left[1-L^{2} \beta_{n}^{2}-2 \beta_{n}\right] \\
& \times\left\|x_{n}-T x_{n}\right\|^{2}+\left(1-\alpha_{n}\right) \delta_{n}\left(\delta_{n}+\gamma_{n}-\beta_{n}\right)\left\|x_{n}-T y_{n}\right\|^{2} \\
& -\left(1-\alpha_{n}\right) \gamma_{n}\left\|x_{n}-T_{\lambda_{n}} x_{n}\right\|^{2}+2 \alpha_{n}\left\langle-x^{*}, w_{n}-x^{*}\right\rangle \\
\leq & \left(1-\alpha_{n}\right)\left\|x_{n}-x^{*}\right\|^{2}+2 \alpha_{n}\left\langle-x^{*}, w_{n}-x^{*}\right\rangle .
\end{aligned}
$$

Now, we consider two cases.

Case 1. Suppose that there exists $n_{0} \in \mathbb{N}$ such that $\left\{\left\|x_{n}-x^{*}\right\|\right\}$ is decreasing for all $n \geq n_{0}$. Then we see that $\left\{\left\|x_{n}-x^{*}\right\|\right\}$ is convergent. Thus, from (3.9) and (3.6) we have

$$
x_{n}-T x_{n} \rightarrow 0, \quad x_{n}-T_{\lambda_{n}} x_{n} \rightarrow 0 \quad \text { as } n \rightarrow \infty .
$$

Moreover, from (3.1) and (3.11) we obtain

$$
\left\|y_{n}-x_{n}\right\|=\beta_{n}\left\|x_{n}-T x_{n}\right\| \rightarrow 0 \quad \text { as } n \rightarrow \infty,
$$

and hence Lipschitz continuity of $T$, (3.12), (3.11) imply that

$$
\begin{aligned}
\left\|T y_{n}-x_{n}\right\| & \leq\left\|T y_{n}-T x_{n}\right\|+\left\|T x_{n}-x_{n}\right\| \\
& \leq L\left\|y_{n}-x_{n}\right\|+\left\|T x_{n}-x_{n}\right\| \rightarrow 0 \quad \text { as } n \rightarrow \infty .
\end{aligned}
$$


In addition, from (3.13) and (3.11) we have

$$
\begin{aligned}
\left\|w_{n}-x_{n}\right\|= & \left\|\left(1-\alpha_{n}\right)\left(\theta_{n} x_{n}+\delta_{n} T y_{n}+\gamma_{n} T_{\lambda_{n}} x_{n}\right)-x_{n}\right\| \\
\leq & \left(1-\alpha_{n}\right) \delta_{n}\left\|T y_{n}-x_{n}\right\|+\left(1-\alpha_{n}\right) \gamma_{n}\left\|T_{\lambda_{n}} x_{n}-x_{n}\right\| \\
& +\alpha_{n}\left\|x_{n}\right\| \rightarrow 0 \quad \text { as } n \rightarrow \infty .
\end{aligned}
$$

Furthermore, since $\left\{w_{n}\right\}$ is bounded subset of $H$ which is reflexive, we can choose a subsequence $\left\{w_{n_{i}}\right\}$ of $\left\{w_{n}\right\}$ such that $w_{n_{i}} \rightarrow w$ and $\lim \sup _{n \rightarrow \infty}\left\langle-x^{*}, w_{n}-x^{*}\right\rangle=\lim _{i \rightarrow \infty}\left\langle-x^{*}, w_{n_{i}}-\right.$ $\left.x^{*}\right\rangle$. It follows from (3.14) that $x_{n_{i}} \rightarrow w$. Then, from (3.11) and Lemma 2.3, we have $w \in F(T)$.

Next, we show that $w \in(A+B)^{-1}(0)$. Let

$$
z_{n}=J_{\lambda_{n}}\left(I-\lambda_{n} A\right) x_{n}
$$

Then from (3.11) we get $z_{n}-x_{n} \rightarrow 0$ as $n \rightarrow \infty$. In addition, for any $p \in \mathcal{F}$, we see that

$$
\begin{aligned}
\left\|z_{n}-p\right\|^{2} & =\left\|J_{\lambda_{n}}\left(I-\lambda_{n} A\right) x_{n}-J_{\lambda_{n}}\left(I-\lambda_{n} A\right) p\right\|^{2} \\
& \leq\left\|x_{n}-p\right\|^{2}-2 \lambda_{n}\left\langle x_{n}-p, A x_{n}-A p\right\rangle+\lambda_{n}^{2}\left\|A x_{n}-A p\right\|^{2} \\
& \leq\left\|x_{n}-p\right\|^{2}-\lambda_{n}\left(2 \gamma-\lambda_{n}\right)\left\|A x_{n}-A p\right\|^{2} .
\end{aligned}
$$

This implies that

$$
\begin{aligned}
\lambda_{n}\left(2 \gamma-\lambda_{n}\right)\left\|A x_{n}-A p\right\|^{2} & \leq\left\|x_{n}-p\right\|^{2}-\left\|z_{n}-p\right\|^{2} \\
& \leq\left(\left\|x_{n}-p\right\|+\left\|z_{n}-p\right\|\right)\left\|x_{n}-z_{n}\right\|,
\end{aligned}
$$

and hence we get

$$
A x_{n}-A p \rightarrow 0 \text { as } n \rightarrow \infty \text {. }
$$

Now from (3.15) we obtain

$$
x_{n_{i}}-\lambda_{n_{i}} A x_{n_{i}} \in\left(I+\lambda_{n_{i}} B\right) z_{n_{i}} .
$$

That is,

$$
\frac{x_{n_{i}}-z_{n_{i}}}{\lambda_{n_{i}}}-A x_{n_{i}} \in B z_{n_{i}} .
$$

Since $B$ is monotone, we get for any $(u, v) \in G(B)$, where $G(B)$ is the graph of $B$ defined by $G(B)=\{(x, w) \in H \times H: x \in D(A), w \in A x\}$,

$$
\left\langle z_{n_{i}}-u, \frac{x_{n_{i}}-z_{n_{i}}}{\lambda_{n_{i}}}-A x_{n_{i}}-v\right\rangle \geq 0
$$

On the other hand, since $\left\langle x_{n_{i}}-w, A x_{n_{i}}-A w\right\rangle \geq \gamma\left\|A x_{n_{i}}-A w\right\|^{2}, x_{n_{i}} \rightarrow w$ and $A x_{n_{i}} \rightarrow A p$, as $n \rightarrow \infty$ we have $A x_{n_{i}} \rightarrow A w$. Thus, letting $i \rightarrow \infty$, we obtain from (3.17)

$$
\langle w-u,-A w-v\rangle \geq 0 .
$$


Thus, maximality of $B$ implies that $-A w \in B w$, that is, $0 \in(A+B)(w)$. Hence, we get $w \in$ $(A+B)^{-1}(0)$.

Therefore, by Lemma 2.1, we immediately obtain

$$
\limsup _{n \rightarrow \infty}\left\langle-x^{*}, w_{n}-x^{*}\right\rangle=\lim _{i \rightarrow \infty}\left\langle-x^{*}, w_{n_{i}}-x^{*}\right\rangle=\left\langle-x^{*}, w-x^{*}\right\rangle \leq 0 .
$$

Then it follows from (3.10), (3.18), and Lemma 2.2 that $\left\|x_{n}-x^{*}\right\| \rightarrow 0$ as $n \rightarrow \infty$. Consequently, $x_{n} \rightarrow x^{*}=P_{\mathcal{F}}(0)$.

Case 2. Suppose that there exists a subsequence $\left\{n_{i}\right\}$ of $\{n\}$ such that

$$
\left\|x_{n_{i}}-x^{*}\right\|<\left\|x_{n_{i}+1}-x^{*}\right\|
$$

for all $i \in \mathbb{N}$. Then, by Lemma 2.4, there exists a nondecreasing sequence $\left\{m_{k}\right\} \subset \mathbb{N}$ such that $m_{k} \rightarrow \infty$, and

$$
\left\|x_{m_{k}}-x^{*}\right\| \leq\left\|x_{m_{k}+1}-x^{*}\right\| \text { and }\left\|x_{k}-x^{*}\right\| \leq\left\|x_{m_{k}+1}-x^{*}\right\| \text {, }
$$

for all $k \in \mathbb{N}$. Now, from (3.9) and (3.6) we get $x_{m_{k}}-T x_{m_{k}} \rightarrow 0$, and $x_{m_{k}}-T_{\lambda_{m_{k}}} x_{m_{k}} \rightarrow 0$ as $k \rightarrow \infty$. Thus, like in Case 1 , we obtain $w_{m_{k}}-x_{m_{k}} \rightarrow 0$ and

$$
\limsup _{k \rightarrow \infty}\left\langle-x^{*}, w_{m_{k}}-x^{*}\right\rangle \leq 0
$$

Now, from (3.10) we have

$$
\left\|x_{m_{k}+1}-x^{*}\right\|^{2} \leq\left(1-\alpha_{m_{k}}\right)\left\|x_{m_{k}}-x^{*}\right\|^{2}+2 \alpha_{m_{k}}\left(-x^{*}, w_{m_{k}}-x^{*}\right\rangle
$$

and hence (3.19) and (3.21) imply that

$$
\begin{aligned}
\alpha_{m_{k}}\left\|x_{m_{k}}-x^{*}\right\|^{2} & \leq\left\|x_{m_{k}}-x^{*}\right\|^{2}-\left\|x_{m_{k}+1}-x^{*}\right\|^{2}+2 \alpha_{m_{k}}\left\langle-x^{*}, w_{m_{k}}-x^{*}\right\rangle \\
& \leq-2 \alpha_{m_{k}}\left\langle x^{*}, w_{m_{k}}-x^{*}\right\rangle .
\end{aligned}
$$

But using the fact that $\alpha_{m_{k}}>0$ and (3.20) we obtain

$$
\left\|x_{m_{k}}-x^{*}\right\| \rightarrow 0 \text { as } k \rightarrow \infty
$$

This together with (3.21) implies that $\left\|x_{m_{k}+1}-x^{*}\right\| \rightarrow 0$ as $k \rightarrow \infty$. But $\left\|x_{k}-x^{*}\right\| \leq \| x_{m_{k}+1}-$ $x^{*} \|$ for all $k \in \mathbb{N}$ and hence we obtain $x_{k} \rightarrow x^{*}$. Therefore, from the above two cases, we can conclude that $\left\{x_{n}\right\}$ converges strongly to the minimum-norm point of $\mathcal{F}$. The proof is complete.

If, in Theorem 3.1, we assume that $A=0$, then we get $T_{\lambda_{n}}\left(x_{n}\right):=\left(I+\lambda_{n} B\right)^{-1} x_{n}$ and hence we get the following corollary.

Corollary 3.2 Let $C$ be a nonempty, closed and convex subset of a real Hilbert space $H$. Let $T: C \rightarrow C$ be a Lipschtzian pseudocontractive mapping with Lipschitz constant $L$ and 
$B: C \rightarrow 2^{H}$ be a maximal monotone mapping. Assume that $\mathcal{F}=F(T) \cap B^{-1}(0)$ is nonempty. Let $\left\{x_{n}\right\}$ be the sequence generated from an arbitrary $x_{0} \in C$ by

$$
\left\{\begin{array}{l}
y_{n}=\left(1-\beta_{n}\right) x_{n}+\beta_{n} T x_{n} ; \\
x_{n+1}=P_{C}\left[\left(1-\alpha_{n}\right)\left(\theta_{n} x_{n}+\delta_{n} T y_{n}+\gamma_{n} T_{\lambda_{n}} x_{n}\right)\right]
\end{array}\right.
$$

where $T_{\lambda_{n}}\left(x_{n}\right):=\left(I+\lambda_{n} B\right)^{-1} x_{n}$ and $\left\{\lambda_{n}\right\} \subset(a, 1),\left\{\theta_{n}\right\},\left\{\delta_{n}\right\},\left\{\gamma_{n}\right\} \subset(c, d) \subset(0,1),\left\{\alpha_{n}\right\} \subset$ $(0, e) \subset(0,1)$, for some $a, c, d, e>0$, satisfying the following conditions: (i) $\theta_{n}+\delta_{n}+\gamma_{n}=1$, (ii) $\lim _{n \rightarrow \infty} \alpha_{n}=0, \sum \alpha_{n}=\infty$; (iii) $\delta_{n}+\gamma_{n} \leq \beta_{n} \leq \beta<\frac{1}{\sqrt{1+L^{2}}+1}, \forall n \geq 1$. Then $\left\{x_{n}\right\}$ converges strongly to the minimum-norm point $x^{*}$ of $\mathcal{F}$.

We also have the following theorem for two maximal monotone mappings.

Theorem 3.3 Let $C$ be a nonempty, closed and convex subset of a real Hilbert space $H$ such that $\operatorname{int}(C) \neq \emptyset$. Let $A, B: C \rightarrow H$ be maximal monotone mappings. Let $T: C \rightarrow C$ be a Lipschitzian pseudocontractive mapping with Lipschitz constant $L$ such that $\mathcal{F}=F(T) \cap$ $(A+B)^{-1}(0)$ is nonempty. Let $\left\{x_{n}\right\}$ be the sequence generated from an arbitrary $x_{0} \in C$ by

$$
\left\{\begin{array}{l}
y_{n}=\left(1-\beta_{n}\right) x_{n}+\beta_{n} T x_{n} \\
x_{n+1}=P_{C}\left[\left(1-\alpha_{n}\right)\left(\theta_{n} x_{n}+\delta_{n} T y_{n}+\gamma_{n} T_{\lambda_{n}} x_{n}\right)\right]
\end{array}\right.
$$

where $T_{\lambda_{n}}\left(x_{n}\right):=\left(I+\lambda_{n}(A+B)\right)^{-1} x_{n}$ and $\left\{\lambda_{n}\right\} \subset(a, 1),\left\{\theta_{n}\right\},\left\{\delta_{n}\right\},\left\{\gamma_{n}\right\} \subset(c, d) \subset(0,1),\left\{\alpha_{n}\right\} \subset$ $(0, e) \subset(0,1)$, for some $a, c, d, e>0$, satisfying the following conditions: (i) $\theta_{n}+\delta_{n}+\gamma_{n}=1$, (ii) $\lim _{n \rightarrow \infty} \alpha_{n}=0, \sum \alpha_{n}=\infty$; (iii) $\delta_{n}+\gamma_{n} \leq \beta_{n} \leq \beta<\frac{1}{\sqrt{1+L^{2}}+1}, \forall n \geq 1$. Then $\left\{x_{n}\right\}$ converges strongly to the minimum-norm point $x^{*}$ of $\mathcal{F}$.

Proof From Lemma 2.7 we find that $A+B$ is a maximal monotone and hence by Corollary 3.2 we get the required assertion.

If, in Theorem 3.3, we assume that $T=I$, the identity mapping on $C$, then we get the following corollary.

Corollary 3.4 Let $C$ be a nonempty, closed and convex subset of a real Hilbert space $H$ such that $\operatorname{int}(C) \neq \emptyset$. Let $A, B: C \rightarrow H$ be maximal monotone mappings such that $\mathcal{F}=$ $(A+B)^{-1}(0)$ is nonempty. Let $\left\{x_{n}\right\}$ be the sequence generated from an arbitrary $x_{0} \in C$ by

$$
x_{n+1}=P_{C}\left[\left(1-\alpha_{n}\right)\left(\left(1-\gamma_{n}\right) x_{n}+\gamma_{n} T_{\lambda_{n}} x_{n}\right)\right] \text {, }
$$

where $T_{\lambda_{n}}\left(x_{n}\right):=\left(I+\lambda_{n}(A+B)\right)^{-1} x_{n}$ and $\left\{\lambda_{n}\right\} \subset(a, 1),\left\{\gamma_{n}\right\} \subset(c, d) \subset(0,1),\left\{\alpha_{n}\right\} \subset(0, e) \subset$ $(0,1)$, for some $a, c, d, e>0$, satisfying the following conditions: $\lim _{n \rightarrow \infty} \alpha_{n}=0$ and $\sum \alpha_{n}=$ $\infty$. Then $\left\{x_{n}\right\}$ converges strongly to the minimum-norm point $x^{*}$ of $\mathcal{F}$.

\section{Applications}

We next study the problem of finding a solution of a variational inequality. Let $C$ be a nonempty closed convex subset of a real Hilbert space $H$. The normal cone for $C$ at a point $x \in C$, denoted by $N_{C}(x)$, is defined by

$$
N_{C}(x)=\left\{x^{*} \in H:\left\langle y-x, x^{*}\right\rangle \leq 0, \forall y \in C\right\} .
$$


Let $f: H \rightarrow(-\infty, \infty]$ be a proper lower semicontinuous convex function. Define the subdifferential

$$
\partial f(x)=\{z \in H: f(x)+\langle y-x, z\rangle \leq f(y), \forall y \in H\}
$$

for all $x \in H$. Then from Rockafellar [38] we know that $\partial f$ is maximal monotone mapping of $H$ into itself. Let $C$ be a nonempty closed convex subset of $H$ and $i_{C}$ be the indicator function of $C$, that is,

$$
i_{C}(x)= \begin{cases}0, & \text { if } x \in C, \\ \infty, & \text { if } x \notin C .\end{cases}
$$

Then $i_{C}: H \rightarrow(-\infty, \infty]$ is a proper lower semicontinuous convex function on $H$ and $\partial i_{C}$ is a maximal monotone mapping. Let $J_{\lambda} x=\left(I+\lambda \partial i_{C}\right)^{-1} x$ for all $\lambda>0$ and $x \in H$. From the fact that $\partial i_{C} x=N_{C} x$ and $x \in C$, we get

$$
\begin{aligned}
u \in J_{\lambda} x & \Leftrightarrow x \in u+\lambda \partial i_{C} u \quad \Leftrightarrow \quad x \in u+\lambda N_{C} u \\
& \Leftrightarrow x-u \in \lambda N_{C} u \quad \Leftrightarrow \quad\langle x-u, y-u\rangle \leq 0, \quad \forall y \in C \\
& \Leftrightarrow u=P_{C} x .
\end{aligned}
$$

Moreover,

$$
\begin{aligned}
x \in\left(A+\partial i_{C}\right)^{-1}(0) & \Leftrightarrow \quad 0 \in\left(A+\partial i_{C}\right) x \quad \Leftrightarrow \quad-A x \in \partial i_{C} x \\
& \Leftrightarrow \quad\langle-A x, y-x\rangle \leq 0, \quad \forall y \in C \\
& \Leftrightarrow \quad x \in \mathrm{VI}(C, A),
\end{aligned}
$$

and hence $x \in\left(A+\partial i_{C}\right)^{-1}(0) \Leftrightarrow x \in \operatorname{VI}(C, A)$. Thus, the following corollary holds. Now, using Theorem 3.1, we obtain a strong convergence theorem for finding a common point of fixed points of Lipschtzian pseudocontractive mapping and solutions of the variational inequality problem for $\gamma$-inverse monotone mapping.

Theorem 4.1 Let $C$ be a nonempty, closed and convex subset of a real Hilbert space H. Let $T: C \rightarrow C$ be a Lipschitzian pseudocontractive mapping with Lipschitz constant $L$ and let $A: C \rightarrow H$ be a $\gamma$-inverse strongly monotone mapping such that $\mathcal{F}=F(T) \cap \operatorname{VI}(C, A) \neq \emptyset$. Let $\left\{x_{n}\right\}$ be the sequence generated from an arbitrary $x_{0} \in C$ by

$$
\left\{\begin{array}{l}
y_{n}=\left(1-\beta_{n}\right) x_{n}+\beta_{n} T x_{n} ; \\
x_{n+1}=P_{C}\left[\left(1-\alpha_{n}\right)\left(\theta_{n} x_{n}+\delta_{n} T y_{n}+\gamma_{n} P_{C}\left(x_{n}-\lambda_{n} A x_{n}\right)\right)\right]
\end{array}\right.
$$

where $\left\{\lambda_{n}\right\} \subset(a, b) \subset(a, 2 \gamma),\left\{\theta_{n}\right\},\left\{\delta_{n}\right\},\left\{\gamma_{n}\right\} \subset(c, d) \subset(0,1),\left\{\alpha_{n}\right\} \subset(0, e) \subset(0,1)$, for some $a, b, c, d, e>0$, satisfying the following conditions: (i) $\theta_{n}+\delta_{n}+\gamma_{n}=1$, (ii) $\lim _{n \rightarrow \infty} \alpha_{n}=0$, $\sum \alpha_{n}=\infty$; (iii) $\delta_{n}+\gamma_{n} \leq \beta_{n} \leq \beta<\frac{1}{\sqrt{1+L^{2}+1}}, \forall n \geq 1$. Then $\left\{x_{n}\right\}$ converges strongly to the minimum-norm point $x^{*}$ of $\mathcal{F}$.

If, in Theorem 4.1, we take $T \equiv I$, the identity mapping on $C$ we have the following corollary for a solution of variational inequality for a $\gamma$-inverse strongly monotone mapping. 
Corollary 4.2 Let $C$ be a nonempty, closed and convex subset of a real Hilbert space $H$. Let $A: C \rightarrow H$ be a $\gamma$-inverse strongly monotone mapping with $\mathrm{VI}(C, A) \neq \emptyset$. Let $\left\{x_{n}\right\}$ be the sequence generated from an arbitrary $x_{0} \in C$ by

$$
x_{n+1}=P_{C}\left[\left(1-\alpha_{n}\right)\left(\left(1-\gamma_{n}\right) x_{n}+\gamma_{n} P_{C}\left(x_{n}-\lambda_{n} A x_{n}\right)\right)\right] \text {, }
$$

where $\left\{\lambda_{n}\right\} \subset(a, b) \subset(a, 2 \gamma),\left\{\gamma_{n}\right\} \subset(c, d) \subset(0,1),\left\{\alpha_{n}\right\} \subset(0, e) \subset(0,1)$, for some a,b,c, $d, e>0$, satisfying the following conditions: $\lim _{n \rightarrow \infty} \alpha_{n}=0, \sum \alpha_{n}=\infty$. Then $\left\{x_{n}\right\}$ converges strongly to the minimum-norm point $x^{*}$ of $\mathrm{VI}(C, A)$.

If, in Theorem 4.1, we take $A:=(I-S)$, where $S$ is a nonexpansive self mapping of $C$ into itself, then we get the following corollary.

Corollary 4.3 Let $C$ be a nonempty, closed and convex subset of a real Hilbert space $H$. Let $T: C \rightarrow C$ be a Lipschitzian pseudocontractive mapping with Lipschitz constant $L$ and let $S: C \rightarrow C$ be a nonexpansive mapping such that $\mathcal{F}=F(T) \cap F(S) \neq \emptyset$. Let $\left\{x_{n}\right\}$ be the sequence generated from an arbitrary $x_{0} \in C$ by

$$
\left\{\begin{array}{l}
y_{n}=\left(1-\beta_{n}\right) x_{n}+\beta_{n} T x_{n} \\
x_{n+1}=P_{C}\left[\left(1-\alpha_{n}\right)\left(\theta_{n} x_{n}+\delta_{n} T y_{n}+\gamma_{n}\left(\left(1-\lambda_{n}\right) x_{n}+\lambda_{n} S x_{n}\right)\right)\right]
\end{array}\right.
$$

where $\left\{\lambda_{n}\right\} \subset(a, b) \subset\left(a, \frac{1}{2}\right),\left\{\theta_{n}\right\},\left\{\delta_{n}\right\},\left\{\gamma_{n}\right\} \subset(c, d) \subset(0,1),\left\{\alpha_{n}\right\} \subset(0, e) \subset(0,1)$, for some $a, b, c, d, e>0$, satisfying the following conditions: (i) $\theta_{n}+\delta_{n}+\gamma_{n}=1$, (ii) $\lim _{n \rightarrow \infty} \alpha_{n}=0$, $\sum \alpha_{n}=\infty$; (iii) $\delta_{n}+\gamma_{n} \leq \beta_{n} \leq \beta<\frac{1}{\sqrt{1+L^{2}}+1}, \forall n \geq 1$. Then $\left\{x_{n}\right\}$ converges strongly to the minimum-norm point $x^{*}$ of $F(T) \cap F(S)$.

Proof Put $A:=I-S$ in Theorem 4.1. Then we see that $A$ is a $\frac{1}{4}$-inverse strongly monotone mapping. Furthermore, for $x \in C$ we have

$$
P_{C}(x-\lambda A x)=P_{C}(x-\lambda(I-T) x)=(1-\lambda) x+\lambda T x
$$

and

$$
\begin{aligned}
x^{*} \in \mathrm{VI}(C, A) & \Leftrightarrow x^{*} \in \mathrm{VI}(C, I-S) \\
& \Leftrightarrow\left\langle S x^{*}-x^{*}, y-x^{*}\right\rangle \leq 0, \quad \forall y \in C \\
& \Leftrightarrow \quad P_{C} S x^{*}=x^{*} \quad \Leftrightarrow \quad S x^{*}=x^{*} .
\end{aligned}
$$

Thus, we obtain $\operatorname{VI}(C, A)=F(S)$. Therefore, the conclusion holds by Theorem 4.1

Remark 4.4 Theorem 3.1 provides convergence sequence to a common point of fixed points of a Lipschitzian pseudocontractive mapping and zeros of two monotone mappings in Hilbert spaces.

Remark 4.5 Theorem 3.1 improves Theorem 3.1 of Takahashi et al. [24] in the sense that our convergence is to the common minimum-norm point of fixed points of a Lipschitzian pseudocontractive mapping and zeros of sum of two monotone mappings. Corollary 3.4 
improves Theorem 1 of Kamimura and Takahashi [14] in the sense that our convergence is for the a zero of sum of two maximal monotone mappings. Theorem 4.1 extends Theorem 3.1 of Takahashi and Toyoda [23] in the sense that our convergence is to the common minimum-norm point of fixed points of a Lipschitzian pseudocontractive mapping and solutions of variational inequality for a $\gamma$-inverse strongly monotone mapping.

\section{Competing interests}

The authors declare that they have no competing interests.

\section{Authors' contributions}

All authors contributed equally to the writing of this paper. All authors read and approved the final manuscript.

\section{Author details}

'Department of Mathematics, King Abdulaziz University, P.O. Box 80203, Jeddah, 21589, Saudi Arabia. ${ }^{2}$ Department of Mathematics, University of Botswana, Pvt. Bag 00704, Gaborone, Botswana.

\section{Acknowledgements}

The authors are grateful to anonymous reviewers for their pertinent remarks and useful suggestions. This article was funded by the Deanship of Scientific Research (DSR), King Abdulaziz University, Jeddah. The first author, therefore acknowledges with thanks DSR for financial support.

Received: 3 January 2014 Accepted: 14 March 2014 Published: 01 Apr 2014

\section{References}

1. Browder, FE, Petryshyn, WV: Construction of fixed points of nonlinear mappings in Hilbert space. J. Math. Anal. Appl. 20, 197-228 (1967)

2. Chidume, CE, Mutangadura, S: An example on the Mann iteration method for Lipschitz pseudocontractions. Proc. Am. Math. Soc. 129, 2359-2363 (2001)

3. Takahashi, W: Nonlinear Functional Analysis. Kindikagaku, Tokyo (1988) (Japanese)

4. Zeidler, E: Nonlinear Functional Analysis and Its Applications. Part II: Monotone Operators. Springer, Berlin (1985)

5. Aoyama, K, Kimura, Y, Takahashi, W, Toyoda, M: On a strongly nonexpansive sequence in Hilbert spaces. J. Nonlinear Convex Anal. 89, 471-489 (2007)

6. Bauschke, $\mathrm{HH}$ : The approximation of fixed points of compositions of nonexpansive mappings in Hilbert spaces J. Math. Anal. Appl. 202, 150-159 (1996)

7. Daman, $\mathrm{OA}$, Zegeye, $\mathrm{H}$ : Strong convergence theorems for a common fixed point of a finite family of pseudocontractive mappings. Int. J. Math. Math. Sci. 2012, Article ID 405315 (2012). doi:10.1155/2012/405315

8. Ishikawa, S: Fixed points by a new iteration method. Proc. Am. Math. Soc. 44, 147-150 (1974)

9. Kamimura, S, Takahashi, W: Strong convergence of proximal-type algorithm in a Banach space. SIAM J. Optim. 13, 938-945 (2002)

10. Yao, YH, Liou, YC, Marino, G: A hybrid algorithm for pseudo-contractive mappings. Nonlinear Anal. 71, 4997-5002 (2009)

11. Zegeye, $\mathrm{H}$, Shahzad, $\mathrm{N}$ : Strong convergence theorems for a common zero of a finite family of $m$-accretive mappings. Nonlinear Anal. 66, 1161-1169 (2007)

12. Rockafellar, RT: Monotone operators and the proximal point algorithm. SIAM J. Control Optim. 14, $877-898$ (1976)

13. Martinet, B: Régularisation d'inéquations variationnelles par approximations successives. Rev. Fr. Inform. Rech. Oper. 4 154-158 (1970)

14. Kamimura, S, Takahashi, W: Approximating solutions of maximal monotone operators in Hilbert spaces. J. Approx. Theory 106, 226-240 (2000)

15. Stampacchia, G: Formes bilineaires coercivites sur les ensembles convexes. C. R. Acad. Sci. Paris 258, 4413-4416 (1964)

16. liduka, H, Takahashi, W: Strong convergence theorems for nonexpansive mappings and inverse-strongly monotone mappings. Nonlinear Anal. 61, 341-350 (2005)

17. Kinderlehrer, D, Stampacchia, G: An Iteration to Variational Inequalities and Their Applications. Academic Press, New York (1990)

18. Yao, Y, Liou, Y-C, Kang, SM: Algorithms construction for variational inequalities. Fixed Point Theory Appl. 2011, Article ID 794203 (2011). doi:10.1155/2011/794203

19. Zegeye, H, Ofoedu, EU, Shahzad, N: Convergence theorems for equilibrium problem, variational inequality problem and countably infinite relatively quasi-nonexpansive mappings. Appl. Math. Comput. 216, 3439-3449 (2010)

20. Zegeye, $\mathrm{H}$, Shahzad, $\mathrm{N}$ : Approximating common solution of variational inequality problems for two monotone mappings in Banach spaces. Optim. Lett. 5, 691-704 (2011)

21. Zegeye, $\mathrm{H}$, Shahzad, N: A hybrid approximation method for equilibrium, variational inequality and fixed point problems. Nonlinear Anal. Hybrid Syst. 4, 619-630 (2010)

22. Zegeye, $\mathrm{H}$, Shahzad, N: Strong convergence theorems for a solution of finite families of equilibrium and variational inequality problems. Optimization 63, 207-223 (2014)

23. Takahashi, W, Toyoda, M: Weak convergence theorems for nonexpansive mappings and monotone mappings. J. Optim. Theory Appl. 118, 417-428 (2003)

24. Takahashi, S, Takahashi, W, Toyoda, M: Strong convergence theorems for maximal monotone operators with nonlinear mappings in Hilbert spaces. J. Optim. Theory Appl. 147, $27-41$ (2010) 
25. Shahzad, N, Udomene, A: Fixed point solutions of variational inequalities for asymptotically nonexpansive mappings in Banach spaces. Nonlinear Anal. 64, 558-567 (2006)

26. Zegeye, H, Shahzad, N: Strong convergence theorems for monotone mappings and relatively weak nonexpansive mappings. Nonlinear Anal. 70, 2707-2716 (2009)

27. Zegeye, $\mathrm{H}$, Shahzad, $\mathrm{N}$ : A hybrid scheme for finite families of equilibrium, variational inequality and fixed point problems. Nonlinear Anal. 74, 263-272 (2011)

28. Yao, Y, Shahzad, N: Strong convergence of a proximal point algorithm with general errors. Optim. Lett. 6, 621-628 (2012)

29. Zegeye, H, Shahzad, N: Viscosity approximation methods for a common fixed point of finite family of nonexpansive mappings. Appl. Math. Comput. 191, 155-163 (2007)

30. Zegeye, $\mathrm{H}$, Shahzad, $\mathrm{N}$ : An algorithm for a common minimum-norm zero of a finite family of monotone mappings in Banach spaces. J. Inequal. Appl. 2013, 566 (2013)

31. Alber, Yl: Metric and generalized projection operators in Banach spaces: properties and applications. In: Theory and Applications of Nonlinear Operators of Accretive and Monotone Type. Lect. Notes Pure Appl. Math., pp. 15-50 (1996)

32. Xu, HK: Another control condition in an iterative method for nonexpansive mappings. Bull. Aust. Math. Soc. 65 109-113 (2002)

33. Zhang, QB, Cheng, CZ: Strong convergence theorem for a family of Lipschitz pseudocontractive mappings in a Hilbert space. Math. Comput. Model. 48, 480-485 (2008)

34. Mainge, PE: Strong convergence of projected subgradient methods for nonsmooth and non-strictly convex minimization. Set-Valued Anal. 16, 899-912 (2008)

35. Zegeye, H, Shahzad, N: Convergence of Mann's type iteration method for generalized asymptotically nonexpansive mappings. Comput. Math. Appl. 62, 4007-4014 (2011)

36. Cai, Y, Tang, Y, Liu, L: Iterative algorithms for minimum-norm fixed point of non-expansive mapping in Hilbert space. Fixed Point Theory Appl. 2012, 49 (2012). doi:10.1186/1687-1812-2012-49

37. Rockafellar, RT: On the maximality of sums of nonlinear monotone operators. Trans. Am. Math. Soc. 194, 75-88 (1970)

38. Rockafellar, RT: On the maximal monotonicity of subdifferential mapping. Pac. J. Math. 33, 209-216 (1970)

$10.1186 / 1687-1812-2014-85$

Cite this article as: Shahzad and Zegeye: Approximating a common point of fixed points of a pseudocontractive mapping and zeros of sum of monotone mappings. Fixed Point Theory and Applications 2014, 2014:85

\section{Submit your manuscript to a SpringerOpen ${ }^{\circ}$ journal and benefit from:}

- Convenient online submission

- Rigorous peer review

- Immediate publication on acceptance

- Open access: articles freely available online

- High visibility within the field

- Retaining the copyright to your article 